\title{
Design and characterization of high-affinity synthetic peptides as bioreceptors for diagnosis of cutaneous leishmaniasis
}

\author{
Y. Andrea Prada ${ }^{1} \cdot$ Maria Soler $^{2,3} \cdot$ Fanny Guzmán ${ }^{4} \cdot$ John J. Castillo $^{5} \cdot$ Laura M. Lechuga $^{2,3} \cdot$ Enrique Mejía-Ospino $^{1}$
}

Received: 7 April 2021 / Revised: 12 May 2021 / Accepted: 20 May 2021 / Published online: 26 May 2021

(C) Springer-Verlag GmbH Germany, part of Springer Nature 2021

\begin{abstract}
Cutaneous leishmaniasis (CL) is one of the illnesses caused by Leishmania parasite infection, which can be asymptomatic or severe according to the infecting Leishmania strain. CL is commonly diagnosed by directly detecting the parasites or their DNA in tissue samples. New diagnostic methodologies target specific proteins (biomarkers) secreted by the parasite during the infection process. However, specific bioreceptors for the in vivo or in vitro detection of these novel biomarkers are rather limited in terms of sensitivity and specificity. For this reason, we here introduce three novel peptides as bioreceptors for the highly sensitive and selective identification of acid phosphatase (sAP) and proteophosphoglycan (PPG), which have a crucial role in leishmaniasis infection. These high-affinity peptides have been designed from the conservative domains of the lectin family, holding the ability to interact with the biological target and produce the same effect than the original protein. The synthetic peptides have been characterized and the affinity and kinetic constants for their interaction with the targets (sAP and PPG) have been determined by a surface plasmon resonance biosensor. Values obtained for $K_{D}$ are in the nanomolar range, which is comparable to high-affinity antibodies, with the additional advantage of a high biochemical stability and simpler production. Pep2854 exhibited a high affinity for sAP $\left(K_{D}=1.48 \mathrm{nM}\right)$ while Pep2856 had a good affinity for PPG $\left(K_{D} 1.76 \mathrm{nM}\right)$. This study evidences that these peptidomimetics represent a novel alternative tool to the use of high molecular weight proteins for biorecognition in the diagnostic test and biosensor devices for CL.
\end{abstract}

Keywords Cutaneous leishmaniasis $\cdot$ High-affinity peptides $\cdot$ Lectins $\cdot$ Proteophosphoglycans $\cdot$ Acid phosphatase

John J. Castillo

jcasleon@uis.edu.co

1 Laboratorio de Espectroscopia Atómica y Molecular (LEAM), Universidad Industrial de Santander, Carrera 27 Calle 9, Bucaramanga, Santander 678, Colombia

2 Networking Research Center on Bioengineering, Biomaterials and Nanomedicine (CIBER-BBN), 28029 Madrid, Spain

3 Nanobiosensors and Bioanalytical Applications Group (NanoB2A), Catalan Institute of Nanoscience and Nanotechnology (ICN2), CSIC, and BIST, Campus UAB, Bellaterra, 08193 Barcelona, Spain

4 Laboratorio de Síntesis de Péptidos, Núcleo de Biotecnología Curauma (NBC), Pontificia Universidad Católica de Valparaíso, 2373223 Valparaíso, Chile

5 Grupo de Investigación en Bioquímica y Microbiología (GIBIM), Universidad Industrial de Santander, Bucaramanga 680002, Colombia

\section{Introduction}

Leishmaniasis, caused by Leishmania parasites, is one of the infectious diseases with the highest incidence in the tropical regions of South America, Africa, the Mediterranean region, and Central Asia, affecting nearly 100 countries. According to the World Health Organization (WHO), there are one million cases of infection each year, including asymptomatic cases which are not reported [1,2]. Leishmaniasis affects the most vulnerable population in the world, usually associated with inadequate nutrition, immunosuppression, and deficiency in the public health system. There are three clinical manifestations of leishmaniasis infection: cutaneous leishmaniasis (CL), mucocutaneous leishmaniasis (ML), and visceral leishmaniasis (VL), known as kala-azar [3]. CL is the most frequent form of the disease; it causes skin lesions that fortunately can heal without treatment in many cases [4]. ML occurs when the parasite spreads into the nose, mouth, and throat producing severe ulcers, destroying the mucous membranes. It can be cured, but the symptoms might not be detected until 
years after infection and, if not treated, it can evolve into serious complications [5]. VL is the most lethal form of the disease; it damages internal organs like spleen or liver, and it affects the bone marrow and the immune system, which results fatal for the patient and death may occur due to complications associated with secondary infections [6].

To date, there is no vaccine available against leishmaniasis. However, a recent study predicted the 3D structure of a vaccine candidate against VL caused by Leishmania donovani based on VC1/VC2 modelled using a molecular dynamics coupled TLR-2 and TLR4 receptors [7]. Therefore, an accurate and early diagnosis of the disease is crucial for the timely administration of treatment and the reduction of the infectious burden $[8,9]$. Importantly, due to the geographic and sociologic distribution of leishmaniasis incidence, it is essential to develop new diagnostic tests that can be implemented as point-of-care (POC) devices and applied for routine screening analysis $[10,11]$. The broad spectrum of clinical manifestations of CL makes its timely diagnosis complicated. Currently, standard methods for the diagnosis of CL include microscopy and microbiological culture of the parasite, which require centralized laboratories, highly specialized personnel, and up to several days for providing conclusive results [12]. The detection of CL via blood serological tests shows a very low sensitivity due to a deficient humoral response by the immune system [13]. This is because the immunological effect in humans is induced by the parasite amastigote form of the parasite (reproductive phase) and not by the promastigote form (infective phase), which infects the macrophage cells immediately after contagion [14]. Clinical assays targeting gene sequences of the parasite using the polymerase chain reaction (PCR) technique have also been developed, as well as immunochemical tests targeting antigens of the cell membrane, such as Kala-Azar Detect and IT-LEISH for VL, based on recombinant Leishmania protein K39, and Montenegro skin test (MTS) for CL $[15,16]$. Other candidates for the detection of leishmaniasis are the membrane glycoproteins gp63, gp70 and gp72, which are specific for the Leishmania genus, and have improved the sensitivity and specificity of ELISA tests [17].

However, these tests are prone to cross-reactions with other diseases caused by trypanosome family members. The limitations of the conventional diagnostic methods are driving research efforts towards the finding of direct biomarkers and potential targets to increase the sensitivity of the diagnosis [18]. Recently, secreted proteins are considered an untapped source of possible antigens and are being exploited using combinations of bioinformatic and immunoproteomics approaches $[19,20]$. Our focus is directed to the glycoproteins secreted during the infectious process by Leishmania parasite, which are promising biomarkers for the detection of CL. Among them, the acid phosphatases (sAP) and proteophosphoglycans (PPG) are secreted by the parasite mostly during the infective phase associated to the promastigote form; therefore, they can play a key critical role as clinical targets for both diagnosis and vaccine development [21].

The sAP are complex enzymes, $\mathrm{N}$-carbohydrate and predominant O-linked phosphorylated complex enzymes, with molecular weight between 90 and $130 \mathrm{kDa}$, which are considered a virulence factors [22]. The sAP inhibits the production of superoxide anions by neutrophils and macrophages, which is closely related to the survival of the parasite inside the host cell. The proteophosphoglycans (PPG), with a molecular weight around $200 \mathrm{kDa}$, are involved in the mammalian infection and may contribute to the binding of Leishmania to the host cell [23].

Nowadays, it is well known that the interaction of mannose-binding lectin with the specific carbohydrates of glycoconjugates on the cell surface of Leishmania acts as an alternative pathway in the complement activation mechanism antibody-independent response [24, 25]. In this sense, lectins can recognize specific glycan arrangements in the PPG molecules [26]. The aim of this work is to design, synthesize, and characterize a new peptide-based galactolectins with enhanced robustness and affinities for both SAP and PPG, to be used for the highly sensitive detection of leishmaniasis [27].

Based on the rational bioinformatic design and theoretical modelling of the interaction, we have obtained three peptide sequences specifically targeting small regions (10-15 amino acid residues) of the lectin domains that can keep the structure-activity relationship. Peptides were synthesized using Fmoc solid-phase peptide chemistry. Synthetic peptides have been fully characterized to determine the binding affinities and kinetic parameters for the interaction with Leishmania PPG and SAP proteins by surface plasmon resonance (SPR) biosensor analysis [28]. SPR is a technique that plays a prominent role in biomolecular interaction characterization due to its ability to detect and monitor molecular binding events in real time without the use of labels. A home-made SPR biosensor working in Kretschmann configuration was employed for the analysis and determination of the binding affinities and kinetic parameters of the interactions between the target proteins and the new peptides. The working principle of the SPR biosensor is based on the high sensitivity of an evanescent plasmonic field generated at the interface of a nanometric metal layer (i.e. $50 \mathrm{~nm}$ of gold) and the dielectric medium when the light strikes at a specific angle. The properties of the evanescent field depend significantly on the refractive index of the medium, whereby the small changes due to molecular interactions can be measured, as well as the formation of molecular pairs (target-bioreceptor) that takes place on the sensor surface. The SPR analysis requires that one of the studied molecules is immobilized on the gold surface chip. Our findings demonstrate the valuable potential of the new 
synthetic peptides as bioreceptors for the direct assay of leishmaniasis biomarkers in diagnostic tests. The higher affinity and stability of our receptors might greatly enhance the analytical performance of conventional clinical assays or innovative diagnostic devices, such as point-of-care biosensors.

\section{Materials and methods}

\section{General chemistry}

All common reagents and materials were obtained from a commercial source such as Sigma-Aldrich and MERCK and used without further purification unless otherwise indicated. Glassware was oven-dried before use and the solutions prepared in deionized water (Resistivity 18.2 $\mathrm{M} \Omega$ ).

\section{Bioinformatic peptide design}

The protein sequences of secreted acid phosphatase and proteophosphoglycans reported by Leishmania ssp. parasites were selected on UniProt Data Bank (Available on: www. uniprot.org/). About twelve sequences of sAP and four sequences of PPG with up to $60 \%$ identity analysed and aligned using both BLAST and Clustal Omega tools (Available on UniProt) for finding a conservative domain in an independent bioinformatic analysis are reported in the Supplementary Information (ESM) Fig. S1a-b. Subsequently, the native models for both sAP and PPG conservative regions were obtained from the GalaxyWEB web server, which allowed making the prediction, refinement, and optimization of the protein structure based on protein template by rescoring HHsearch results. Then a group of 15 sequences of glycoconjugate-recognized lectins were selected from Protein Data Bank and ten fragment peptides between 14 and 18 amino acid residues of different motif regions of the proteins which were extracted from Protein Data Bank (https://www.rcsb.org/) with ID codes: 1CIW; ICQ9; ICR7; IQF3; 1RIR; 2DHI; 2DV9; 2DVA;2DVB;2DVD; 2DVF; 2DVG; 2DVP; 2TEP; 1BZW. The secondary structure conformation of each peptide was modelled and visualized using the PEPFOLD server and PyMOL v2.3 respectively. The experimental data suggest that three peptide sequences described as Pep2854, Pep2855, and Pep2856 adopted a conformational space-optimized based on high interaction similarity with the targets.

\section{Theoretical modelling of peptide-glycoprotein interactions}

CABS-dock server (http://biocomp.chem.uw.edu.pl/ CABSdock) was used to investigate the protein-peptide interaction. For the performance of the dynamic simulation, the algorithms found the binding site with full spatial flexibility of peptide and decreased the fluctuation on the protein backbone without an a priori knowledge of the binding site [29, 30]. To evaluate the results, the RMSD value was used, which is defined as the root mean square deviation of the peptideprotein interaction. For selecting the better models, the contact maps were calculated with a cut-off distance value of $4.5 \AA$ for each peptide-glycoconjugate complex (ESM Fig. S3a-b).

\section{Synthesis and purification of peptides}

Pep2854 (HYSRTENMGTSNAC), Pep 2855 (K T F L K I C K R P Y V P S E P), and Pep 2856 (INKQGDVTVLSNGNTQLT) were synthesized by solidphase peptide synthesis following the Fmoc methodology. Briefly, peptide building blocks were loaded on of Rink amide 4MBHA resin ( $0.55 \mathrm{mmol} / \mathrm{g}$ substitution; $100 \mathrm{mg}$ per tea bag) followed by chain linkage via the coupling of Fmoc-protected L-amino acids between the C-terminal residue and the resin. The amino acids were coupled using a mixture of the L-amino acid, O-(Benzotriazol-1-yl)-N,N, $\mathrm{N}^{\prime}, \mathrm{N}^{\prime}$-tetramethyluronium hexafluorophosphate (HBTU), or oxime and $\mathrm{N}$ ethyldiisopropylamine (DIEA) in the ratio 5:5:5:7.5, respectively, for over $3 \mathrm{~h}$. The Fmoc protecting groups were removed with piperidine $(20 \% \mathrm{v} / \mathrm{v}$ in $0.01 \%$ Tween 20 solution). All coupling reactions were carried out in dimethylformamide. The peptides were cleaved and the lateral chain protecting groups were removed using a cocktail containing trifluoroacetic acid (TFA), triisopropylsilane (TIS), and water (ratio 95:2.5:2.5). Lastly, the peptides were precipitated with cold diethyl ether, centrifuged thrice, and the pellet was dissolved in water before lyophilization. The crude peptides were purified by RP-HPLC on a Zorbax C-18 preparative column $(7.0 \mu \mathrm{m} ; 21.5 \times 250 \mathrm{~mm})$ using a gradient from 30 to $70 \%$ of acetonitrile (ACN) (Mobil phase A: $\mathrm{H}_{2} \mathrm{O}$, $0.001 \%$ TFA, and B: ACN, $0.001 \%$ TFA).

\section{Mass spectrometry analysis}

The fractions collected from RP-HPLC were analysed by MALDI-TOF spectrometry in an UltrafleXtreme Bruker Daltonics mass spectrometer using a dried-droplet method using an $\alpha$-cyano-4-hydroxycinnamic acid saturated solution $\left(0.001 \%\right.$ TFA in $\left.\mathrm{ACN} / \mathrm{H}_{2} \mathrm{O}\right)$ as matrix substance for MALDI.

\section{Circular dichroism analysis}

The secondary structure peptide structures were analysed by circular dichroism in a Jasco J-815 spectropolarimeter with an acquisition speed of $20 \mathrm{~nm} / \mathrm{min}$ in a spectral region of 190 $260 \mathrm{~nm}$. 


\section{Surface plasmon resonance biosensor}

SPR experiments were performed at room temperature $\left(25^{\circ} \mathrm{C}\right)$ on a home-made SPR biosensor. The sensor platform is based on the Kretschmann configuration that allowed the monitoring of the spectrum of the reflected light at $70^{\circ}$ of incident angle on a 50-nm layer of the gold sensor chip, previously biofunctionalized with each peptide. The biosensor surface is clamped in a flow cell and is irradiated with a halogen lamp (HL-2000, Ocean Optics, USA) in transverse electric polarization mode; the light passes through a trapezoidal prism for plasmonic excitation. Then, the reflected light is collected by an optical fibre coupled to a CCD spectrometer (Ocean Optics-Jazz Module, USA). The microfluid delivery system includes a syringe pump (New Era, NE1000, USA), connected to the flow cell. Samples of sAP and PPG were manually injected using an injection valve (IDEZ Health and Science, V-451, USA). Thereby, the biomolecular interactions that take place on the sensor surface can be detected by the changes in the refraction index and the displacement of the plasmon wavelength $\left(\Delta \lambda_{\mathrm{SPR}}\right)$. Data are presented as a sensorgram where the $\Delta \lambda_{\mathrm{SPR}}$ is evaluated in real time.

\section{Sensor surface biofunctionalization}

The sensor chip is based on an ultra-thin gold layer ( $50 \mathrm{~nm}$ thickness) deposited on a glass slide previously covered with titanium (2.0 nm thickness). The sensor chips were washed with acetone, isopropanol, and Milli-Q water by immersing at boiling temperature. After that, they were dried with $\mathrm{N}_{2}$ and placed in an ozone cleaner (BioForce Nanoscience, USA) for $40 \mathrm{~min}$. A self-assembled monolayer (SAM) was carried out ex situ using a mixture of functional alkanethiols, mercaptohexadecanoic acid (MHDA), and mercaptoundecanol (MUOH) in a 1:1 ratio and total concentration of $250 \mu \mathrm{M}$ in ethanol, where the sensor chips were incubated overnight. Then, the nanoplasmonic sensors were rinsed with ethanol and dried with $\mathrm{N}_{2}$. The carboxylic group activation was performed via carbodiimide ester formation using a mixture of 1-ethyl-3-(3-dimethylaminopropyl)carbodiimide (EDC) and Nhydroxysuccinimide ester (NHS) $(0.2 \mathrm{M} / 0.05 \mathrm{M})$ solution in 2-(N-morpholino)ethanesulfonic acid (MES) buffer (100 mM; pH 6.8) for $30 \mathrm{~min}$. Afterward, each peptide (Pep2854, Pep2855, and Pep2856) was covalently immobilized on a gold sensor chip using a $50 \mu \mathrm{g} \mathrm{mL}^{-1}$ peptide solution in MES buffer (50 mM; pH 7.0) by incubation overnight to ensure a high density of bioreceptor on the sensor chip surface. Finally, ethanolamine (1 M; $\mathrm{pH}$ 8.5) was added to deactivate the unreactive carboxylic group. The sensor chips were rinsed with Milli-Q water and dried with $\mathrm{N}_{2}$.

\section{Binding interaction analysis on sensor chips}

The dilution series of targets were injected (sAP and PPG) in separate experiments for each peptide without exceeding the saturation concentration of the analyte onto the sensor chip surface. In the case of sAP, the range of concentrations injected was from 5.2 to $104 \mathrm{nM}$ in $10 \mathrm{mM}$ MES buffer $\mathrm{pH}$ 6.5. Moreover, the PPG was injected in concentrations between 3.0 and $58 \mathrm{nM}$ prepared in $10 \mathrm{mM}$ acetate buffer $\mathrm{pH}$ 4.5. The running buffer for each assay was the same as for the sample to avoid a bulk refractive index effect, and the flow rate was $30 \mu \mathrm{L} / \mathrm{min}$ applied for $15 \mathrm{~min}$. The regeneration step was carried out so that all peptides were able to dissociate entirely using $5.0 \mathrm{mM}$ of $\mathrm{NaOH}$ at a flow rate of $30 \mu \mathrm{L} / \mathrm{min}$ for 2 min as shown in Fig. S6 (see ESM). The sensorgrams acquired were fitted to a one-site binding interaction model using GraphPad Prism software (Version 9.0). This model assumed that the formation of the target-ligand complex is not limited by mass transport because the SPR biosensor can keep a constant target concentration in a continuous flow. Therefore, in the kinetic analysis, the constants of association $\left(\mathrm{k}_{\mathrm{on}}\right)$ and dissociation $\left(\mathrm{k}_{\mathrm{off}}\right)$ were calculated from the plot of $\Delta \lambda_{\text {SPR }}$ vs concentration [28]. Consequently, the equilibrium dissociation constant $\left(\mathrm{K}_{\mathrm{D}}\right)$, known as affinity, was determined from the relation $K_{D}=k_{o f f} / k_{\text {on }}$. Additionally, the $\operatorname{chi}^{2}$ value was reported as a parameter indicative of the fitting confidence.

For the determination of SPR sensitivity, we used buffer PBS serial solutions prepared in Milli-Q water; then refraction index of each PBS solution was measured in a refractometer and was injected in order of less to major concentration. Then $\Delta \lambda$ was determined from the LSPR vs real-time graphical as shown in the Fig. S4 of ESM.

\section{Results and analysis}

\section{Design and synthesis of specific PPG and SAP bioreceptor peptides}

We have developed three new synthetic peptides for the sensitive and selective detection of PPC and SAP proteins, secreted by the Leishmania parasite during the infection process. The rational design of peptides was based on the conservative domains of lectins specific to cell surface carbohydrates as the Gal-Man-(PO4) type and on the analysis of the active sites which were previously identified and reported on the Protein Data Bank (PDB). The finding conserved domains in sAP and PPG are able to predict the interaction between peptides and these biomolecules which will mostly take place in these regions increasing the probability that the designed peptides can recognize any SAP or PPG secreted by a broad range number of Leishmania species. Therefore, from the Clustal Omega 
results of the sAP secreted by Leishmania reported in UniProt, we selected the region corresponding to MVQVVHRHGARSALNDNTTEICGTLYPC (Code Job UniProt: /www.uniprot.org/align/A20190117216DA2B77 BFBD2E6699CA9B6D1C41EB202DE21L), and for PPG, we chose the region corresponding to $1030-1450$ of the proteophosphoglycan with A4HM87 as the more conservative region by the only four sequences reported (Code Job Uniprot: w w w . uniprot.org/a lign/A 20190918674 6803381A1F0E0DB47453E0216320D05A710T.aln). The PDB codes, Clustal Omega, and the BLAST results are included in Fig. S1a-b of the ESM.

The peptides were synthesized and purified using Sepack C-18 columns, and the secondary structure was analysed by circular dichroism as shown in Fig. 1. The structure of a lectin is reported in the Protein Data Bank with PDB code 2DHI. This protein belonging to this family is characterized by showing mostly both beta-folded and $\alpha$-helix motifs in theirs structures. In addition, the peptides retain the conformation, which is a very important observation considering that peptides having less than 20 amino acids are crucial in the active design of functional peptides that can access to a specific biological target. The Pep2854 and Pep2855 exhibited a strong tendency to a $\alpha$-helix structure because of the presence of amino acids such as His, Cys, Glu, Asp, and Ala, while Pep2856 showed a ß-sheet profile, favoured by the presence of Gly, Val, Ile, and Leu residues (Fig. 2b-c). Our experimental data support the results of the theoretical folding of the most likely conformation peptides performed on the PEPFOLD server.
This finding gives excellent reliability and robustness to the molecular docking between these peptides and the glycoproteins under study. Bo et al. show that the peptide sequences of proteins often have intrinsic propensities for the formation of their native conformations [30] which is inconsistent with our designed peptide that keeps the motifs structural from lectins.

The most relevant physicochemical properties (i.e. molecular weight, net charge, isoelectric point, etc.) of the peptidomimetics are presented in Table 1. As for the molecular dynamics performed, the CABS-dock server-generated ten top-ranked model structures of both the sAPpeptide and the PPG-peptide complex. The docking poses predicts the binding orientations of a ligand to the receptor active site, many times defined by the user to evaluate stabilizing interactions such as hydrogen bonds, salt bridges, and hydrophobic contacts possible in the pairs of molecules using PDB files [31]. The computational experiment allowed the full flexibility of the novel synthesized peptides and a small fluctuation of the receptor backbone to explore the binding position according to the score function energies by calculating the root mean square deviation (RMSD) of each pose [32].

Three different RMSD classifications for docking solutions were used as a reference: (i) right solution when RMSD $\leq$ $2.0 \AA$; (ii) acceptable solution when RMSD is between 2.0 and $4.0 \AA$; and (iii) wrong solution when RMSD $\geq 4.0 \AA$ [33]. The present work was oriented by prior knowledge $[34,35]$ of the interactions that may take place between three
Fig. 1 Circular dichroism spectra of the tendency of secondary structures of each synthetic peptide in $30 \% \mathrm{v} / \mathrm{v}$ in trifluoroethanol with a peptide concentration of $0.1 \mathrm{mg} \mathrm{mL}^{-\mathbf{1}}$. a Secondary structure of a lectin reported with PDB code: 2 DHI. b Pep2854 2D-folding with an $\alpha$ helix structure tendency. c Pep2855 2D folding with an $\alpha$ helix structure stronger tendency. d Pep2856 2D folding with a beta-sheet tendency $\mathbf{a}$
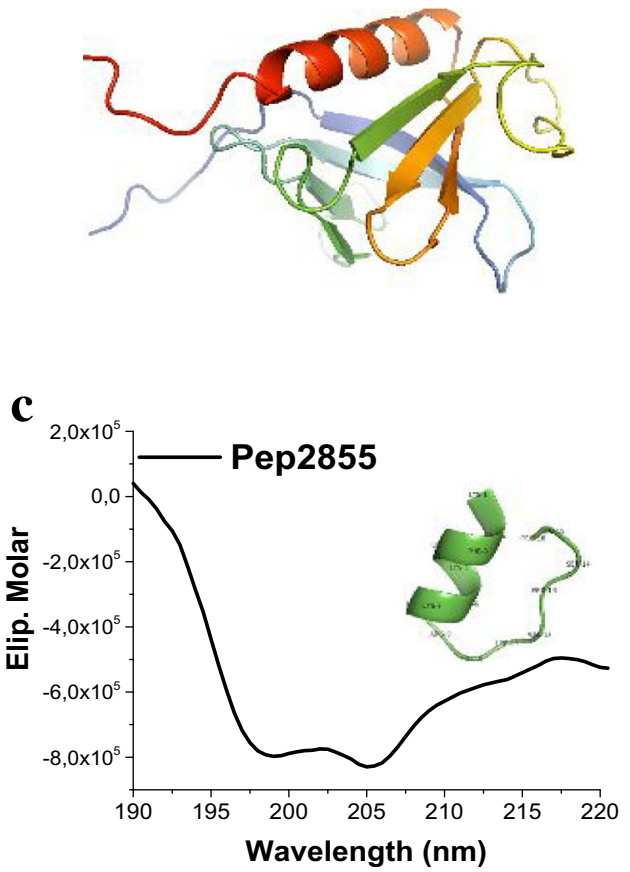

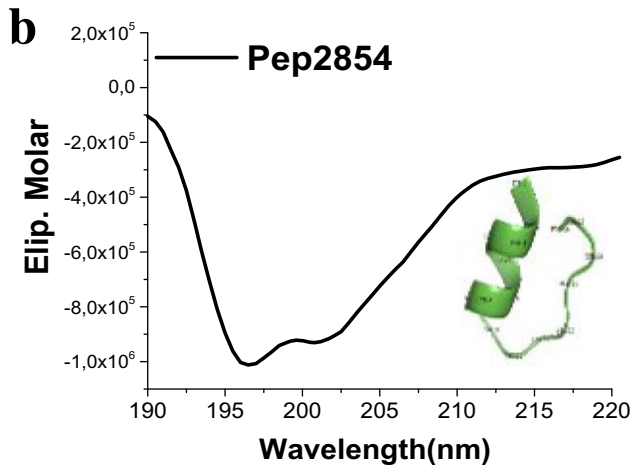

d

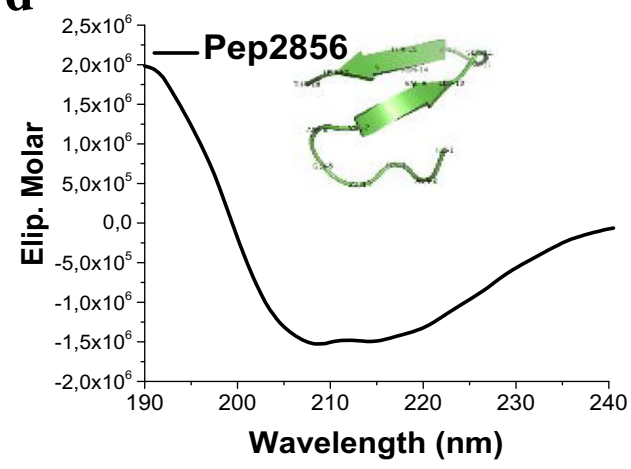




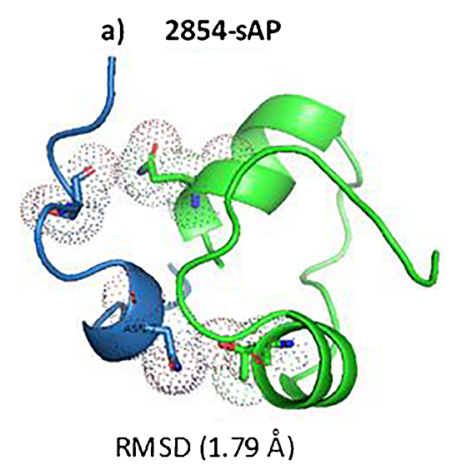

f) 2854-PPG

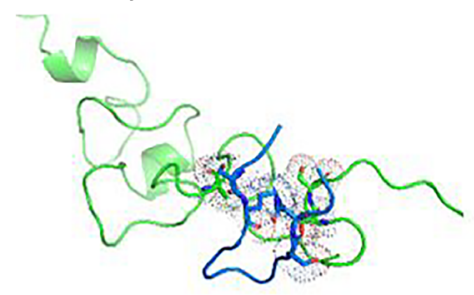

$\operatorname{RMSD}(6.54 \AA$ )

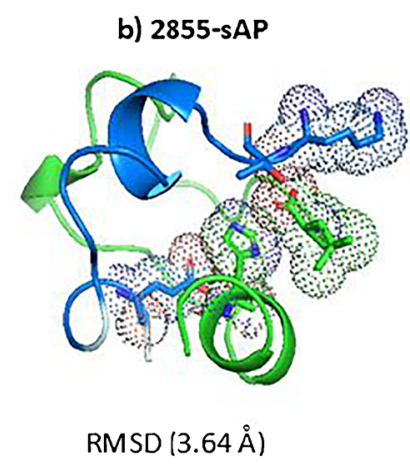

e) 2855-PPG

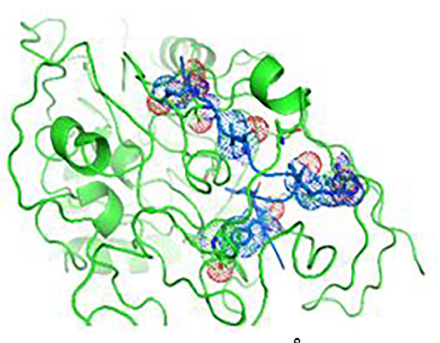

$\operatorname{RMSD}(3.48 \AA)$

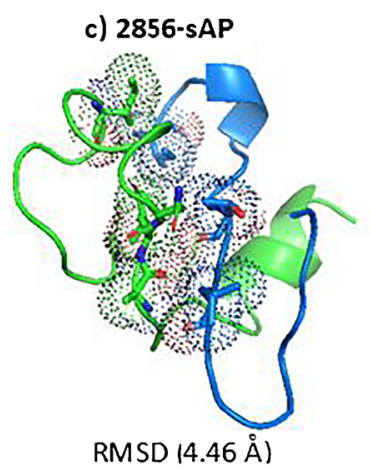

d) 2856-PPG

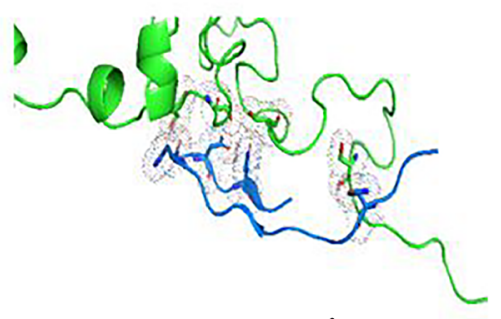

$\operatorname{RMSD}(7.51 \AA)$
Fig. 2 Top-ranked models obtained from CABS-dock docking with the peptide-RMSD values. The structures represent each of the peptides bound close to a SAP fragment of the conservative region corresponding to MVQVVHRHGARSALNDNTTEICGTLYPC $(\mathbf{a}-\mathbf{c})$. The structures represent each of the peptides bound close to the PPG binding at the conservative region (UniProt code: A4HM87 ${ }_{1030-1450}$ ) (d-f). The target region of glycoprotein is coloured in green, and the peptide is coloured in blue. The dotted region represents the most likely site for binding interaction according to the contact map peptides homologous to lectin and two different glycoconjugates. As shown in Fig. 2, the partial loss of the local structure conformation of the peptides can be seen when they are approaching the sAP region. The more frequent contacts were between sAP residues Arg11, Ser 12, and Asn17, and Pep2854 residues Asn7, Ser11, and Asn11 at a highfrequency level (0.06-0.1) according to the contact maps.

For the dynamic molecular trajectories, the smallest RMSD of atomic coordinate value was $1.79 \AA$ in the docking for trajectories of the Pep2854-sAP pair, thus validating the structure-based design process. We focused on the clustering based on the fewer RMSD values released to the peptide bond in the proximity of the binding site on the bioreceptor. For PPG in Fig. 2, it was hypothesised that Ser275, Ser289, and Ser363 of the proteophosphoglycan domain are the key binding sites to the cationic peptides through Lys, Arg, and Glu residues, since in this case most interactions were salt bridges.
For Pep2855-PPG molecular dynamics, the smallest calculated RMSD value was $3.48 \AA$; this value is on the borderline of a bad result. However, this must be considered since the calculation of RMSD increased significantly with the molecular size of the MD structures; then, the RMSD became a data with many attributes when the high dimensionality causes the algorithms based on the clustering methods to calculate the trajectory poses to fail [36]. Hence, these data are considered as good approximations, which were complemented and correlated with the affinity counters obtained by subsequent SPR experiments.

\section{Determination of binding affinities by surface plasmon resonance biosensor analysis}

The strategy used was the formation of the covalent amine coupling between the N-hydroxy succinimide (NHS-
Table 1 Physicochemical properties of synthetic peptides designed from lectin mannosegalactose-binding proteins

\begin{tabular}{lllcccc}
\hline ID peptide & Sequence & MW (Da) & $\mathrm{C}^{*}$ & $\mathrm{pI}$ & 2D folding & \#AA \\
\hline Pep2854 & HYSRTENMGTSNAC & 1569.68 & +1 & 8.58 & Alpha-helix & 14 \\
Pep2855 & KTFLKICKRPYVPSEP & 1905.31 & +4 & 10.5 & Alpha-helix & 16 \\
Pep2856 & INKQGDVTVLSNGNTQLT & 1901.08 & +1 & 10.14 & Beta-sheet & 18 \\
\hline
\end{tabular}

*Net charge at $\mathrm{pH} 7.0$; isoelectric point (pI); number of amino acidic residues (AA) 
1. MHDA:MUOH [1:1]Overnight

2. EDC:NHS in buffer MES pH 6.8

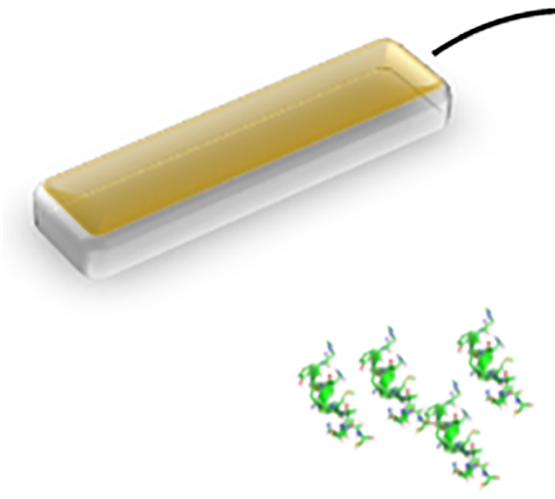

3. Peptide $\left[50 \mu \mathrm{g}-\mathrm{mL}^{-1}\right]$ Overnight 4. Ethanolamine

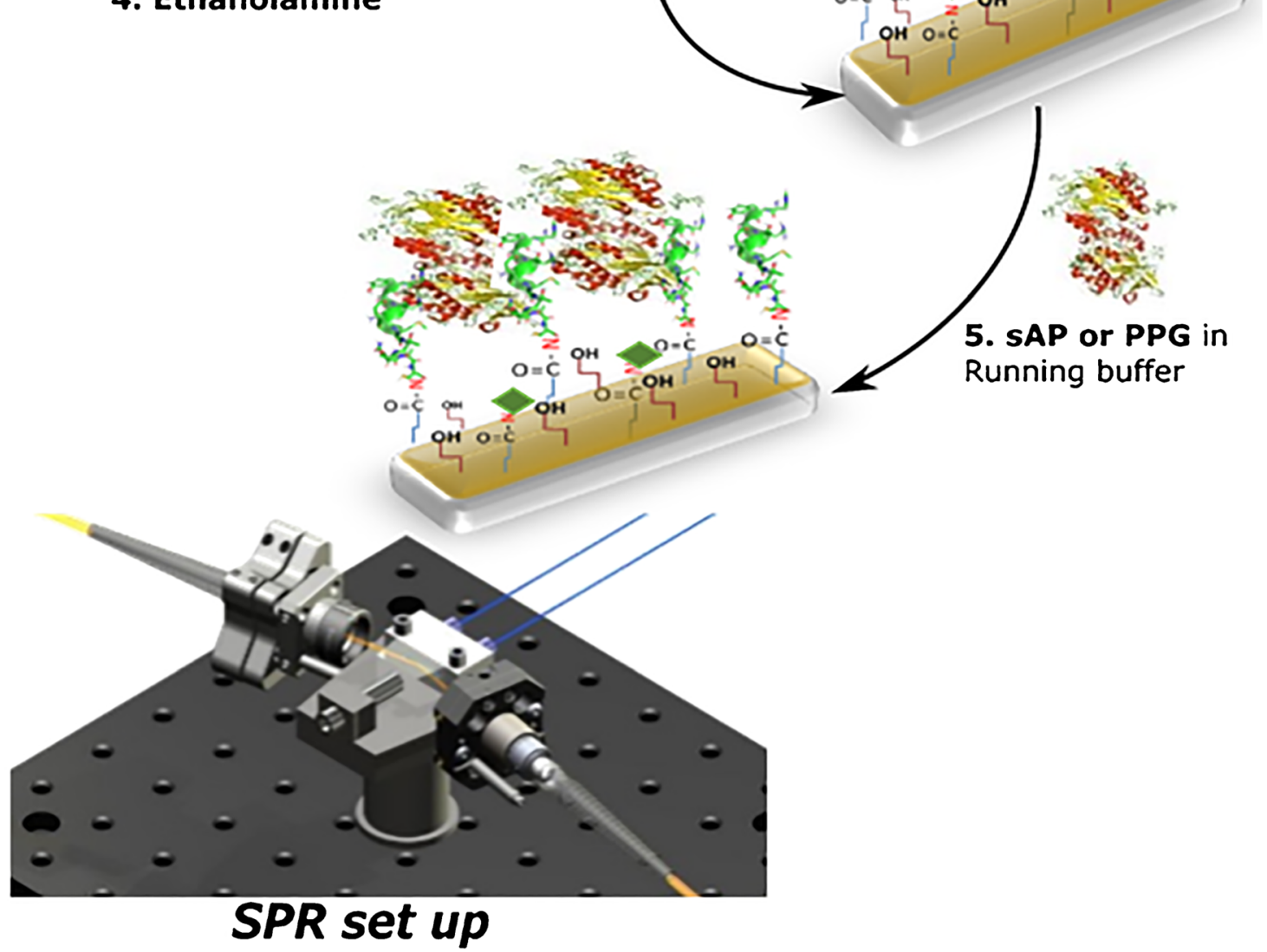

Fig. 3 Biofunctionalization strategy onto a plasmonic sensor chip for SPR analysis of the binding interaction between each of the synthetic peptides with both sAP and PPG

activated) of a $\mathrm{COOH}$-functional alkanethiol self-assembled monolayer (SAM) and the primary amine group present in each peptide chain [37] (see Fig. 3). To facilitate the homogeneous distribution of the peptide monolayer on the surface and minimize steric hindrance issues, hydroxyl-functional alkanethiols were used as a lateral spacer in the SAM.

We independently evaluated the interaction of sAP and PPG with the three peptidomimetics, previously immobilized on the sensor chip. Results shown in Fig. 4 correspond to the fitting of the experimental sensorgram to the theoretical kinetic parameters and the statistical parameter for all fitted curves (chi and R-square) 12.

The sensorgrams resulting from the injection of the sAP and PPG solution over the SAM without immobilized peptide showed the full recovery of the baseline after the injection of the targets, confirming the absence of nonspecific adsorptions to the sensor surface at the best conditions of peptide immobilization (ESM Fig. S2). From the fitting equations in Tables 2 and 3, it is possible to extract both kinetics $\left(\mathrm{k}_{\mathrm{on}}\right.$ and $\left.\mathrm{k}_{\mathrm{off}}\right)$ and affinity parameters for the interaction. In terms of 

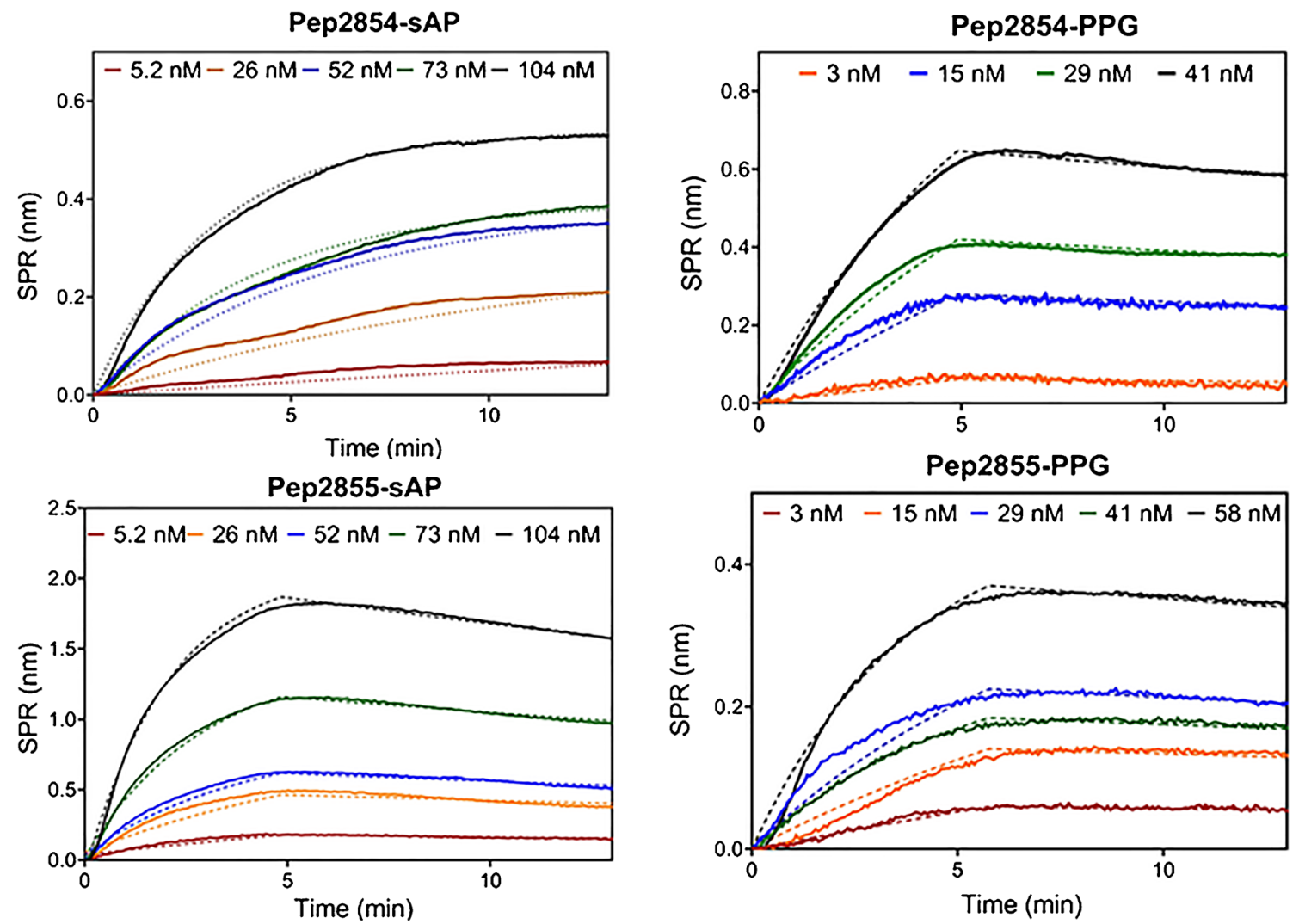

Pep2856-sAP
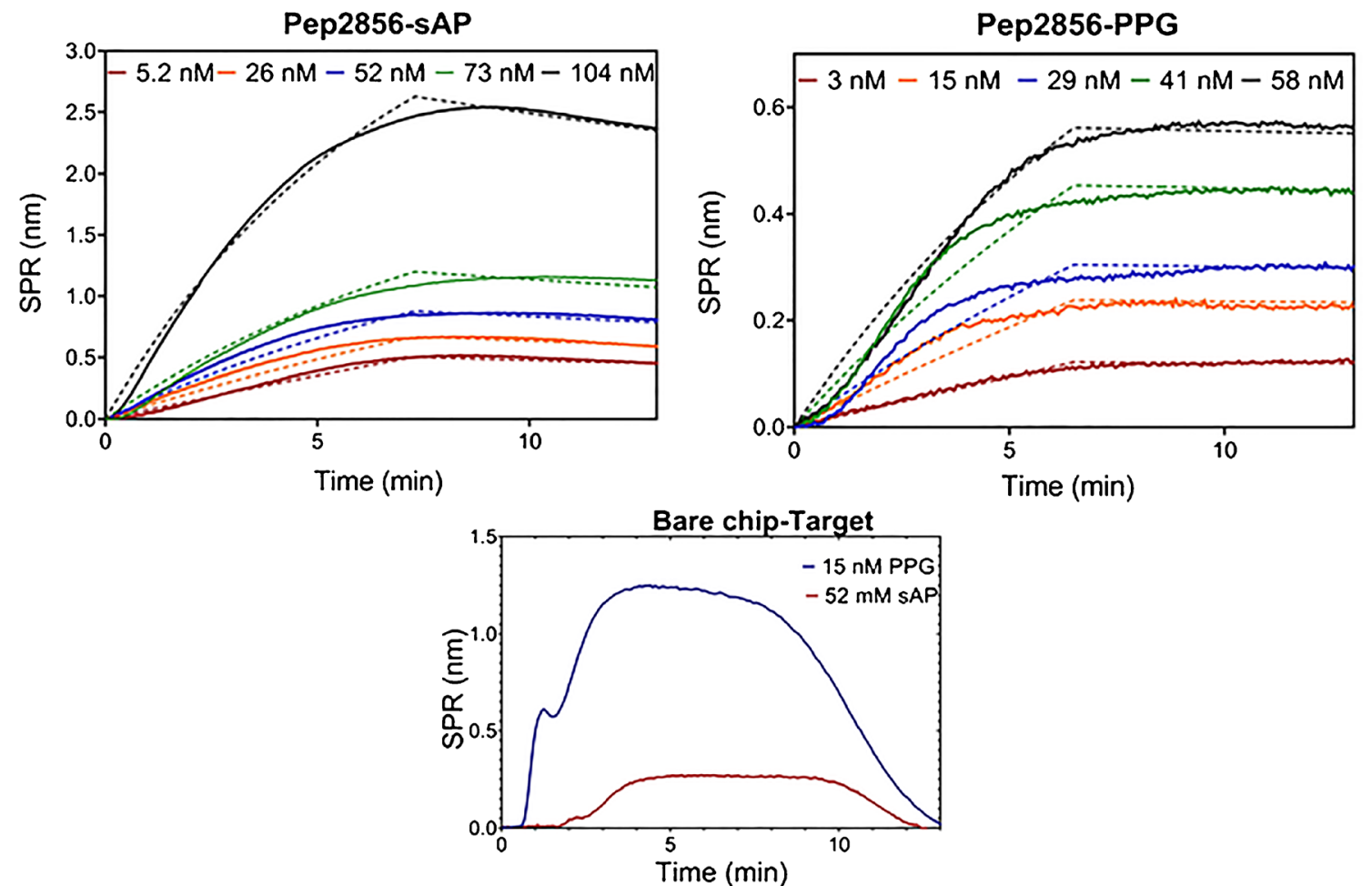

Fig. 4 SPR analysis of peptide interactions with both SAP and PPG. The SAP was tested in the concentration range from 5.2 to $104 \mathrm{nM}$, while for $\mathrm{PPG}$, the concentration range was from 3.0 to $58 \mathrm{nM}$, enough to cover the kinetic curves. The sensorgrams are shown in continuous lines, while fitting curves are shown in dotted line. The figure included a sensorgram of the interaction of sAP and PPG with the sensor surface without immobilized peptide 
Table 2 Kinetic parameters of the interaction of synthetic peptides with sAP

\begin{tabular}{llllll}
\hline ID peptide & $\mathrm{K}_{\mathrm{D}}(\mathrm{nM}) \pm \mathrm{SE}$ & $\mathrm{k}_{\text {on }}\left(\times 10^{6} \mathrm{M} \mathrm{s}^{-1}\right) \pm \mathrm{SE}$ & $\mathrm{k}_{\text {off }}\left(\mathrm{s}^{-1}\right) \pm \mathrm{SE}$ & $\mathrm{R}$-square $\left(\mathrm{R}^{2} \approx 1\right)$ & Chi $($ chi $\approx 0)$ \\
\hline Pep2854 & $1.48 \pm 0.10$ & $3.15 \pm 0.23$ & $0.004 \pm 0.001$ & 0.995 & 0.012 \\
Pep2855 & $4.42 \pm 0.09$ & $4.49 \pm 0.63$ & $0.019 \pm 0.002$ & 0.997 & 0.025 \\
Pep2856 & $15.6 \pm 0.49$ & $1.26 \pm 0.30$ & $0.020 \pm 0.003$ & 0.995 & 0.044 \\
\hline
\end{tabular}

* SE, standard error of the mean. $\mathrm{K}_{\mathrm{D}}$ was estimated based on both steady-state affinity and kinetic steady-state 1:1 interaction model from three independent experiments kinetics, the association constant $\left(\mathrm{k}_{\mathrm{on}}\right)$ indicates the rapidness of the biomolecular interaction but, importantly, the dissociation constant $\left(\mathrm{k}_{\mathrm{off}}\right)$ is a measure of the stability of the complex. In this sense, the Pep2854-sAP and Pep2856-PPG complexes are the most stable ones and their $\mathrm{k}_{\text {off }}$ dissociation constants showed small values of 0.004 and $0.001 \mathrm{~s}^{-1}$, respectively. This observation can be interpreted as the fraction of the complex that dissociates every second, which is related to a longer half-life of these two molecular complexes. Regarding the $\mathrm{k}_{\mathrm{on}}$ measurement, we observed that Pep2855 quickly bounds to the two target molecules, showing similar $\mathrm{k}_{\mathrm{on}}$ values for both $\operatorname{sAP}\left(4.49 \times 10^{-6} \mathrm{M} \mathrm{s}^{-1}\right)$ and PPG $\left(4.4 \times 10^{-6} \mathrm{M} \mathrm{s}^{-1}\right)$; however, these interactions were not the most stable. We infer from this finding that both Pep2854 and Pep2856 not only have a high affinity for the studied glycoproteins but also exhibit a distinct specificity for one of the targets. Affinity is the binding strength of a molecule to its ligand, and even though binding is a reversible process, the measurement of the reaction rate constants can be used to define an equilibrium or affinity constant $\left(1 / K_{D}\right)$, so the smaller the $K_{D}$ value, the higher the affinity of the peptide for its target [37].

All peptides showed $K_{D}$ values in the nanomolar range, evidencing the high affinity for sAP and PPG. The molecular pairs, Pep2854-sAP and Pep2856-PPG, registered the smallest $\mathrm{K}_{\mathrm{D}}$ values equal to $1.48 \mathrm{nM}$ and $1.76 \mathrm{nM}$, respectively, as shown in Tables 2 and 3. One of the most significant observation is that the interaction of the Pep2856-PPG was 9fold stronger than Pep2856-sAP, presumably because the positive charge of Pep2856 (+4) increases the electrostatic interaction with the negative groups in the PPG molecule at sites different to the active site. These affinity values are like the $K_{D}$ values of high-affinity antibodies, generally considered to be in the range of 1 to $10^{-3} \mathrm{nM}$ [38]. Our results demonstrated than the affinity values are similar to the $K_{D}$ values reported by other research where many peptide-based protein ligands, called synbodies, have high affinity and with a $\mathrm{K}_{\mathrm{D}}<5 \mathrm{nM}[39$, 40]. Nevertheless, synthetic peptides show some advantages over antibodies, such as their simple and robust production at large scales and their high biochemical stability, therefore offering an interesting alternative for their use in diagnostic tests and devices. Finally, we demonstrated the specificity of the peptides towards the molecules tested. The interaction by the peptides is not significant since an increase in the signal is observed due to changes in the refractive index of the medium due to the bulky effect of BSA when it passes through the sensor surface (see ESM Fig. S5).

\section{Conclusions}

We have designed and synthesized three high-affinity peptides based on the galactolectin family, which can recognize Leishmania SAP and PPG proteins, considered as potential targets for the diagnosis of the infection. The dynamic molecular strategy employed allowed to model the most likely interactions between peptides and the targets selected, finding RMSD values within the acceptable limits for theoretical calculations ( $3.0 \AA$ ). Pep2854 and Pep2856 exhibited the highest affinity and specificity for sAP $\left(K_{D}\right.$ of $\left.1.48 \mathrm{nM}\right)$ and PPG $\left(K_{D}\right.$ of $1.76 \mathrm{nM})$. These results support the hypothesis that peptides based on a rational design with the ability to occupy the binding site of a target glycoprotein can be used as high-affinity bioreceptors, being an alternative to large biomolecules as antibodies with limitations in terms of production, biochemical stability, and robustness. These peptides can be employed in the development of new diagnostic tools for cutaneous leishmaniasis, especially for the construction of point-of-care
Table 3 Kinetic parameters of the interaction of synthetic peptides with PPG

\begin{tabular}{lrllll}
\hline ID peptide & $\mathrm{K}_{\mathrm{D}}(\mathrm{nM}) \pm \mathrm{SE}$ & $\mathrm{k}_{\text {on }}\left(\times 10^{6} \mathrm{M} \mathrm{s}^{-1}\right) \pm \mathrm{SE}$ & $\mathrm{k}_{\text {off }}\left(\mathrm{s}^{-1}\right) \pm \mathrm{SE}$ & R-square $\left(\mathrm{R}^{2} \approx 1\right)$ & Chi $(\mathrm{chi} \approx 0)$ \\
\hline Pep2854 & $5.0 \pm 0.26$ & $2.6 \pm 0.10$ & $0.014 \pm 0.005$ & 0.995 & 0.014 \\
Pep2855 & $2.7 \pm 0.11$ & $4.4 \pm 0.09$ & $0.012 \pm 0.003$ & 0.993 & 0.008 \\
Pep2856 & $1.76 \pm 0.50$ & $1.8 \pm 0.01$ & $0.001 \pm 0.002$ & 0.987 & 0.019 \\
\hline
\end{tabular}

*SE, standard error of the mean. $\mathrm{K}_{\mathrm{D}}$ was estimated based on both steady-state affinity and kinetic steady-state 1:1 interaction model from three independent experiments 
biosensor devices that enable rapid and accurate detection of the infection in decentralized and low-resource settings.

Supplementary Information The online version contains supplementary material available at https://doi.org/10.1007/s00216-021-03424-2.

Acknowledgements We thank Patricia Escobar from CINTROP for helpful discussion about Leishmania parasite.

Funding This study was supported by a Doctoral Fellowship from COLCIENCIAS in Colombia. The ICN2 is funded by the CERCA programme/Generalitat de Catalunya. ICN2 is supported by the Severo Ochoa program from Spanish Ministry of Science MICINN (Grant No. SEV-2017-0706). This work has made use of the Biodeposition and Biodetection Unit from ICTS NANBIOSIS (http://www.nanbiosis.es/ portfolio/u4-biodeposition-andbiodetection-unit/) partially supported by MICINN/FEDER (FICTS-1420-27).

\section{Declarations}

Conflict of interest The authors declare no competing interests.

\section{References}

1. Ejov M, Dagne D. Strategic framework for leishmaniasis control in the WHO European Region 2014-2020.; 2014.

2. Melkamu HT, Beyene AM, Zegeye DT. Knowledge, attitude and practices of the resident community about visceral leishmaniasis in West Armachiho district, Northwest Ethiopia. Heliyon. 2020;6(1): e03152. https://doi.org/10.1016/j.heliyon.2019.e03152.

3. Alvar J, Vélez ID, Bern C, et al. Leishmaniasis worldwide and global estimates of its incidence. PLoS One. 2012;7(5). https:// doi.org/10.1371/journal.pone.0035671.

4. Scott $P$, Novais FO. Cutaneous leishmaniasis: immune responses in protection and pathogenesis. Nat Rev Immunol. 2016;16(9):58192. https://doi.org/10.1038/nri.2016.72.

5. Crone CG, Helleberg M. Cutaneous leishmaniasis with secondary mucosal disease in a traveller due to Leishmania (Viannia) braziliensis. J Travel Med. 2020;27(1):1-4. https://doi.org/10. 1093/jtm/taz093.

6. Elmahallawy EK, Sampedro Martínez A, Rodriguez-Granger J, et al. Diagnosis of leishmaniasis. J Infect Dev Ctries. 2014;8(8): 961-72. https://doi.org/10.3855/jidc.4310.

7. Singh G, Pritam M, Banerjee M, Singh AK, Singh SP. Designing of precise vaccine construct against visceral leishmaniasis through predicted epitope ensemble: a contemporary approach. Comput Biol Chem. 2020;86(December 2019):107259. https://doi.org/10. 1016/j.compbiolchem.2020.107259.

8. Savoia D. Recent updates and perspectives on leishmaniasis. J Infect Dev Ctries. 2015;9(6):588-96. https://doi.org/10.3855/jidc. 6833.

9. Can H, Döșkaya M, Özdemir HG, et al. Seroprevalence of Leishmania infection and molecular detection of Leishmania tropica and Leishmania infantum in stray cats of I'zmir, Turkey. Exp Parasitol. 2016;167:109-14. https://doi.org/10.1016/j.exppara. 2016.05.011.

10. Soler M, Huertas CS, Lechuga LM. Label-free plasmonic biosensors for point-of-care diagnostics: a review. Expert Rev Mol Diagn. 2019;19(1):71-81. https://doi.org/10.1080/14737159.2019. 1554435 .
11. Ashrafmansouri MA, Sarkari B, Hatam G, Habibi P, Abdolahi Khabisi S. Utility of western blot analysis for the diagnosis of cutaneous leishmaniasis. Iran J Parasitol. 2015;10(4):599-604.

12. de Paiva-Cavalcanti M, de Morais RCS, Pessoa-e-Silva R, et al. Leishmaniases diagnosis: an update on the use of immunological and molecular tools. Cell Biosci. 2015;5(1):1-10. https://doi.org/ 10.1186/s13578-015-0021-2.

13. Romero GAS, Orge MDLGO, Guerra MVDF, Paes MG, Macêdo VDO, De Carvalho EM. Antibody response in patients with cutaneous leishmaniasis infected by Leishmania (Viannia) braziliensis or Leishmania (Viannia) guyanensis in Brazil. Acta Trop. 2005;93(1):49-56. https://doi.org/10.1016/j.actatropica.2004.09. 005 .

14. Soulat D, Bogdan C. Function of macrophage and parasite phosphatases in leishmaniasis. Front Immunol. 2017;8(DEC):1-21. https://doi.org/10.3389/fimmu.2017.01838.

15. Reiter-Owona I, Rehkaemper-Schaefer C, Arriens S, Rosenstock P, Pfarr K, Hoerauf A. Specific K39 antibody response and its persistence after treatment in patients with imported leishmaniasis. Parasitol Res. 2016;115(2):761-9. https://doi.org/10.1007/s00436015-4801-8.

16. Herrera G, Castillo A, Ayala MS, Flórez C, Cantillo-Barraza O, Ramirez JD. Evaluation of four rapid diagnostic tests for canine and human visceral Leishmaniasis in Colombia. BMC Infect Dis. 2019;19(1):1-9. https://doi.org/10.1186/s12879-019-4353-0.

17. Ibarra-Meneses AV, Moreno J, Carrillo E. New strategies and biomarkers for the control of visceral leishmaniasis. Trends Parasitol. 2020;36(1):29-38. https://doi.org/10.1016/j.pt.2019.10.005.

18. Kip AE, Balasegaram M, Beijnen JH, Schellens JHM, De Vries PJ, Dorloa TPC. Systematic review of biomarkers to monitor therapeutic response in leishmaniasis. Antimicrob Agents Chemother. 2015;59(1):1-14. https://doi.org/10.1128/AAC.04298-14.

19. Bahrami F, Harandi AM, Rafati S. Biomarkers of cutaneous leishmaniasis. Front Cell Infect Microbiol. 2018;8(JUN):1-8. https:// doi.org/10.3389/fcimb.2018.00222.

20. Esteves S, Costa I, Amorim C, Nuno Santarem AC-S. Biomarker indicator of abnormal physicological process. Biomarkers in leishmaniasis: from basic research to clinical application. 2018:195224. https://doi.org/10.1016/j.colsurfa.2011.12.014.

21. Pinedo-Cancino V, Laurenti MD, Kesper N, Umezawa ES. Evaluation of Leishmania (Leishmania) infantum excretedsecreted antigens for detection of canine leishmaniasis. Acta Trop. 2016;161:41-3. https://doi.org/10.1016/j.actatropica.2016. 05.0100 .

22. Ejazi SA, Bhattacharyya A, Choudhury ST, et al. Immunoproteomic identification and characterization of Leishmania membrane proteins as non-invasive diagnostic candidates for clinical visceral leishmaniasis. Sci Rep. 2018;8(1):1-11. https://doi.org/10.1038/s41598-018-30546-y.

23. Tham M, Ramasamy S, Gan HT, et al. CSPG is a secreted factor that stimulates neural stem cell survival possibly by enhanced EGFR signaling. PLoS One. 2010;5(12). https://doi.org/10.1371/ journal.pone.0015341.

24. Pelletier I, Hashidate T, Urashima T, et al. Specific recognition of Leishmania major poly- $\beta$-galactosyl epitopes by galectin-9: possible implication of galectin-9 in interaction between L. major and host cells. J Biol Chem. 2003;278(25):22223-30. https://doi.org/ 10.1074/jbc.M302693200.

25. Padilla-Docal B, Dorta-Contreras A, Bu-Coifiu R, Callol-Barroso J. Rol de la lectina de unión a manosa en infecciones parasitarias. Rev Panam Infecto. 2009;11(3):45-8.

26. Pustylnikov S, Sagar D, Jain P, Khan ZK. Targeting the C-type lectins-mediated host-pathogen interactions with dextran. J Pharm Pharm Sci a Publ Can Soc Pharm Sci Soci??t?? Can des Sci Pharm. 2014;17(3):371-92. 
27. Blaszczyk M, Kurcinski M, Kouza M, et al. Modeling of proteinpeptide interactions using the CABS-dock web server for binding site search and flexible docking. Methods. 2016;93:72-83. https:// doi.org/10.1016/j.ymeth.2015.07.004.

28. Sparks RP, Jenkins JL, Fratti R. Use of surface plasmon resonance (SPR) to determine binding affinities and kinetic parameters between components important in fusion machinery. Methods Mol Biol. 1860;2019:199-210. https://doi.org/10.1007/978-1-49398760-3_12.

29. Kurcinski M, Pawel Ciemny M, Oleniecki T, Kuriata A, Badaczewska-Dawid AE, Kolinski A, et al. CABS-dock standalone: a toolbox for flexible protein-peptide docking. Bioinformatics. 2019;35:4170-2. https://doi.org/10.1093/ bioinformatics/btz185.

30. Ho BK, Dill KA. Folding very short peptides using molecular dynamics. PLoS Comput Biol. 2006;2(4):228-37. https://doi.org/10. 1371/journal.pcbi.0020027.

31. Hua C, Hui X, Zhu L, Chang S, Zu W, Xin C. Complex-typedependent scoring functions in protein - protein docking 2007;129:1-10. doi:https://doi.org/10.1016/j.bpc.2007.04.014.

32. Watkins AM, Bonneau R, Arora PS. Highly flexible proteinpeptide docking using CABS-Dock. Methods Mol Biol. 2017;1561:109-38. https://doi.org/10.1007/978-1-4939-6798-8.

33. Sargsyan K, Grauffel C, Lim C. How molecular size impacts RMSD applications in molecular dynamics simulations. J Chem Theory Comput. 2017;13(4):1518-24. https://doi.org/10.1021/acs. jctc. $7 \mathrm{~b} 00028$.

34. Li J, Wu H, Hong J, Xu X, Yang H, Wu B, et al. Odorranalectin is a small peptide lectin with potential for drug delivery and targeting.
PLoS One. 2008;3(6):1-10. https://doi.org/10.1371/journal.pone. 0002381.

35. Arnaud J, Audfray A, Imberty A. Binding sugars: from natural lectins to synthetic receptors and engineered neolectins. Chem Soc Rev. 2013;42(11):4798-813. https://doi.org/10.1039/ c2 $\operatorname{cs} 35435 \mathrm{~g}$.

36. Yap BK, Leung EWW, Yagi H, et al. A potent cyclic peptide targeting SPSB2 protein as a potential anti-infective agent. J Med Chem. 2014;57(16):7006-15. https://doi.org/10.1021/jm500596j.

37. Monfregola L, Vitale RM, Amodeo P, De Luca S. A SPR strategy for high-throughput ligand screenings based on synthetic peptides mimicking a selected subdomain of the target protein: a proof of concept on HER2 receptor. Bioorg Med Chem. 2009;17(19):701520. https://doi.org/10.1016/j.bmc.2009.08.004.

38. Wang Y, Zhang X, Xie Y, Wu A, Zai X, Liu X. High-affinity phage-displayed peptide as a recognition probe for the detection of Cry2Ad2-3. Int J Biol Macromol. 2019;137:562-7. https://doi. org/10.1016/j.ijbiomac.2019.06.164.

39. Diehnelt CW, Shah M, Gupta N, Belcher PE, Greving MP, Stafford $\mathrm{P}$, et al. Discovery of high-affinity protein binding ligands - backwards. PLoS One. 2010;5. https://doi.org/10.1371/journal.pone. 0010728.

40. Xiao W, Ma W, Wei S, Li Q, Liu R, Carney RP, et al. High-affinity peptide ligand LXY30 for targeting $\alpha 3 \beta 1$ integrin in non-small cell lung cancer. J Hematol Oncol. 2019;12:1-18. https://doi.org/10. 1186/s13045-019-0764-z.

Publisher's note Springer Nature remains neutral with regard to jurisdictional claims in published maps and institutional affiliations. 